PoS $\quad \begin{aligned} & \text { PROCEEDINGS } \\ & \text { OF SCIENCE }\end{aligned}$

\title{
A life spent for the research in nuclear science and.....
}

Roberto BASSINI*

INFN- Sezione di Milano, Via Celoria 16, I-20133 Milano (Italy)

E-mail: bassiniemi.infn.it

XLVIII International Winter Meeting on Nuclear Physics in Memoriam of Ileana Iori 25-29 January 2010

Bormio, Italy

${ }^{*}$ Speaker. 


\section{Introduction}

With the present paper I intend to emphasise the success related to Ileana' s great idea of strongly involving the Electronic Laboratory and the Workshop of Milan in experiments requiring new and cutting edge electronics developments. It is thanks to this idea that we grew very much during the years and we were able to become an important Team for experiments in Heavy Ions Physics. In addition to myself, the team included Ciro Boiano, Augusto Bassi, Sergio Brambilla, Massimo Malatesta, the support of Angelo Cortesi from the machine shop and also students working in detector constructions.

All began in 1980 with the first experiments at LNL with the Bragg ionization chambers and continued with the design and the construction of the following detectors:

- Multi-detectors systems (MULTICS) - GANIL, MSU and LNS (since 1993);

- Catania Hodoscopes - GSI, MSU, LNS (since 1996);

- Time-of-Flight of HADES - GSI (since 1998).

In the following a brief description of selected most relevant devices designed and constructed during many years of very productive and rewarding activity is given.

\section{The MULTICS array}

MULTICS [1] is an array of 56 telescopes (see Fig. 1), covering the forward angular region from about $3^{\circ}$ to about $28^{\circ}$ with a geometric efficiency of $72 \%$, designed to detect light charged particles and heavy ions at intermediate energies. It has been widely used in experiments at GANIL (Caen, France) and at the NSCL facility of MSU (East Lansing, USA).

Each element of the array is a four-fold telescope made up by an axial ionization chamber, a $5 \times 5$ $\mathrm{cm}^{2}$ (500 mm thick) two-dimensional position-sensitive Si diode, a $3.5 \mathrm{~mm} \mathrm{Si}(\mathrm{Li})$ detector and a $2.5 \mathrm{~cm}$ CsI crystal, read out by a photodiode. After MULTICS, the Si-CsI(Tl) coupling became the typical telescope in heavy-ion physics.

The angular resolution is about $0.1^{\circ}$ in the central part $\left(3 \times 3 \mathrm{~cm}^{2}\right)$ of each detector and about $0.3^{\circ}$ near the edges; charge identification has been proven to extend up to $\mathrm{Z}=83$ and the isotope separation up to oxygen ions: it is a detection system that combines a large dynamical range with good charge, energy and angular resolutions.

The signals from the detectors are handled by means of standard electronics chains for the pulse height analysis, based on charge preamplifier (PA), spectroscopy amplifier (SA) and analog to digital converter (ADC). A PA sensitivityof $1 \mathrm{mV} / \mathrm{MeV}$ ( $\mathrm{Si}$ ) is used for the silicon signals while 45 or $90 \mathrm{mV} / \mathrm{MeV}$ ( $\mathrm{Si}$ ) are used for the IC and PD signals. Particular care has been devoted, in the development of the board that allocates the preamplifiers, to assure no detectable crosstalk.

The amplifiers are built as four-fold units; the coarse and fine gains of any individual channel are controlled by a VME central unit. Shaping timesof $1.5 \mu$ s and $3 \mu$ s are used for the IC, Si and PD respectively. The signals from the silicon anodes and the photodiodes are also handled by fast amplifiers followed by constant fraction discriminators to generate triggers and set the bit-pattern modules. 


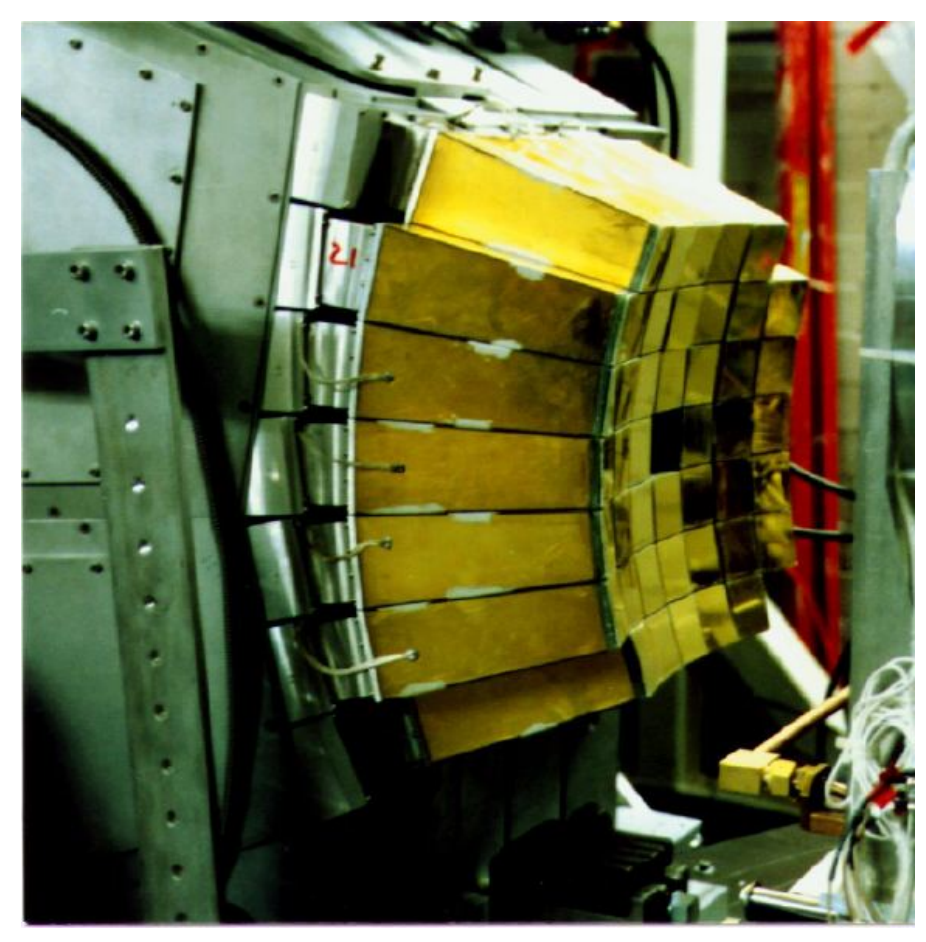

Figure 1: The Multics detector array.

The main amplifiers, the ADC and the TDC as well as the PA, have been expressively developed by the Laboratory of Electronics of the Milano Nuclear Division and are commercially available. In particular the 4 Channel Spectroscopy Amplifier Fully Programmable [3] and the 4 Channel ADC on a VME Board [4] have initiated and pushed the designs of completely programmable boards with consistent increment number of channels.

The ADC in particular was the first important module designed in standard VME having performances comparable with the commercial ADCs in standard NIM usually utilized in that period in the heavy-ion experiments.

\section{The Catania Hodoscopes}

The Si-CsI hodoscopes are multi-detectors arrays designed for coincidence measurements. They were widely used on a variety of experiments performed at GSI (Darmstadt, Germany) [5], at NSCL-MSU (East Lansing, USA) and at LNS-Catania, aiming at the study of hot nuclear matter and nuclei far from stability [6].

These arrays consist on double and triple-stage telescopes with silicons detectors followed by $\mathrm{CsI}(\mathrm{Tl})$ crystals with photodioed readout. The signals from each detector are handled by means of a standard electronics chain for pulse- height analysis: it is based on charge preamplifier (PA), shaping amplifier (SA), stretcher and analog-to-digital converter (QDC and TDC).

Preamplifiers, with a sensitivity of $45 \mathrm{mV} / \mathrm{MeV}$, are used for both the silicon and the photodiode signals. They are mounted in boxes, holding two motherboards each, with twelve 8-fold sections housing up to 96 elements. Each section has an independent power-supply input ( \pm 12 and \pm 24 


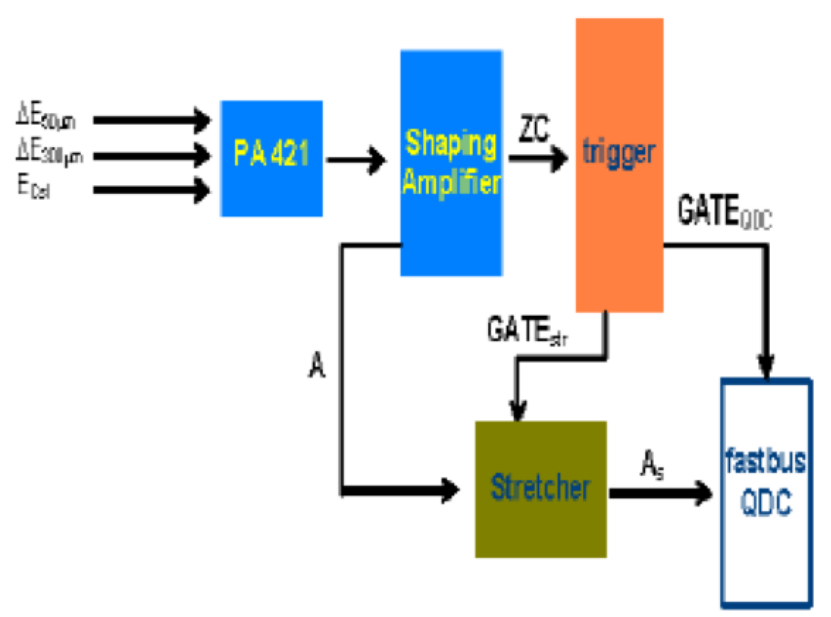

Figure 2: Schematic view of the electronics associated to each telescope.

Volt), whereas common test-pulse and bias supply inputs are partitioned in parallel.

In order to reduce overheating of the preamplifiers, which operate in vacuum inside the scattering chamber, a cooling system was realized, equipping each box with a brass piece, machined in order to contain pipeline for the cooling fluid coming from the cooling machine.

Preamplifiers signals are shaped with 8-folds shaping amplifiers. For each channel three values of shaping time can be chosen: $0.5,1$ and $3 \mu \mathrm{sec}$.

Each channel of the eight gives an analogical output in a lemo connector and leading-edge (LE) and zero-crossing (Z.C.) logical signals, grouped in a two-fold 8-pin ECL connector. Shaping time of 1.5 and $3 \mu \mathrm{sec}$ have been used for Si and CsI detectors respectively.

The Z.C. signals are sent to the AND-OR-ECL modules. Each of these modules has 32 logic ECL inputs and provides the logical OR of all channel in a two-fold 16 pin ECL output and the analog output of the total multiplicity ( $150 \mathrm{mV}$ per fired channel).

The analog outputs of the shapers are then sent to stretchers: they need a common gate input signal derived from the trigger, that fix the time interval in which stretching of the incoming signals at their maxima occurs (followed then by a Reset signal). These modules accept negative-polarity input signals with a frequency below $63.3 \mathrm{kHz}$ and an amplitude between $8 \mathrm{mV}$ and $8 \mathrm{~V}$.

Analog signals coming from the stretchers are finally integrated and digitized by a 96-channel Fastbus LeCroy QDC with a common gate also derived from the trigger.

In Fig. 2 a schematic view of the electronic chain used for processing the signals coming from each telescope is shown. Also in the case of this detector arrays, the whole front-end electronics was on purpose designed by our Laboratory in Milan in close collaboration with the University and INFN in Catania.

\section{Hybrid low-noise charge preamplifiers for heavy-ion detectors}

The know-how acquired setting up the above mentioned detectors motivated further studies on the development of low-noise charge preamplifiers (see Fig. 3) to be used in heavy-ion experi- 


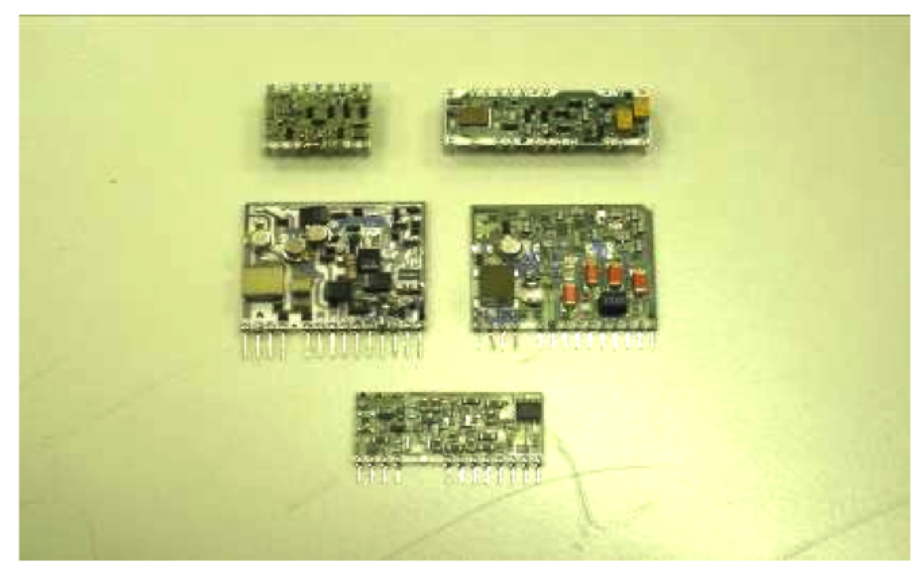

Figure 3: Various hybrid low-noise charge preamplifiers bulit between 1982 and 2008.

ments [2]

Indeed the electronic handling of the signals from these detectors requires charge-sensitive preamplifiers, essentially based on an operational integrator giving an output voltage signal proportional to the charge collected by a feedback capacitor. These units must match the characteristics of each detector as well as the constraints of the experimental conditions. As normally the detector arrays are very compact, the preamplifiers must have small dimensions and a low power consumption. They have to be placed as close to the detectors as possible.

These conditions suggest the choice of thick-film hybrid technology, which offers the advantages of the high packing density in small geometries, the possibility to obtain reliable devices in large quantity and low component cost. The entire circuit is mounted on one side of an alumina frame while the back side is metalized and grounded. Anywhere possible, the ground connections have been obtained reaching theback side through holes made with a laser beam. Special care has been devoted to minimizing the power consumption and to assuring stable performances. Eventually, this last goal has been achieved by taking advantage of the good electrical properties and the high resistance to moisture of an acrylic coating film.

As homogeneity of the response functions of the large number of preamplifiers required by the apparatus is highly desirable, special attention has been addressed to the values of the feedback and the test-input capacitors: the nominal value has been obtained, with a precision better than $1 \%$, laser cutting one electrode of these capacitors.

Taking into account, for the different detectors, the energy losses of the significant ions and the energy required to create an ion pair, the preamplifiers have been designed to have different energy sensitivities.

A particular problem in the preamplifier design is related to the position sensitivity of the silicon detectors. As the position information is obtained through charge division with a resistive chain, either continuous or discrete, an input resistance not very low (say $1 \mathrm{k} \Omega$ ) can spoil most of the position resolution, even if the preamplifier performs satisfactorily when only the energy signal is required .

Finally, the detectors of each telescope differ in the value of the dark current: itr anges from essentially zero for the ionization chamber anode to a few $\mu \mathrm{A}$ for the large-area silicon detectors. 


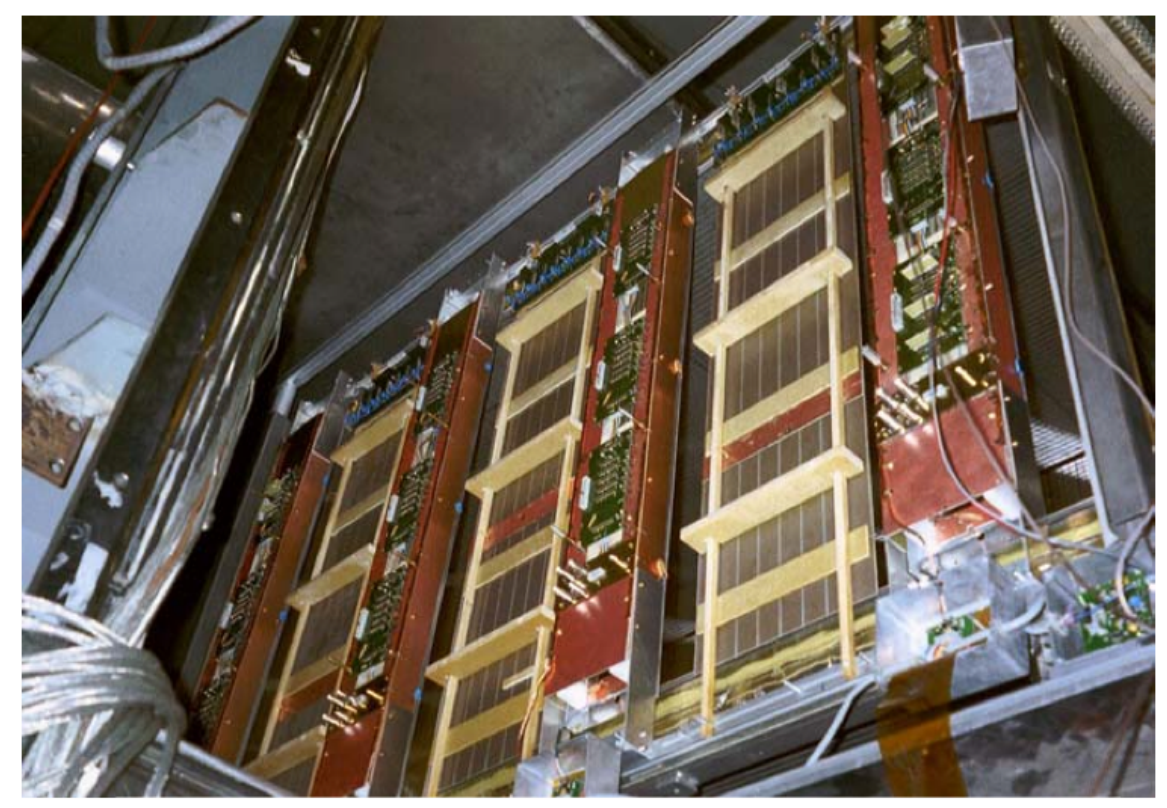

Figure 4: Side view of the TP-MUSIC detector.

It is obvious that the high value of the load resistor, appropriate for the first case, can prevent the possibility of applying the necessary bias voltage to the silicon detectors. Thus the hybrid circuit board provides the possibility to easily connect, in parallel to the factory-mounted $100 \mathrm{M} \Omega$ resistor, a user-selected load resistor.

Lately a new model of these preamplifiers has been succesfully used on a new generation TimeProjection-Chamber at GSI (the TP-MUSIC IV detector [7]).

It's a tracking ionisation chamber. The detector can detect nuclei from $\mathrm{He}$ up to $\mathrm{Au}$. Because the energy loss of a charged particle is proportional to the square of its charge, the detector should, with the associated readout electronics, be able to collect signals extending over a wide dynamic range. In order to achieve for light and also heavy fragments the best possible resolution the TPMUSIC detector has two different types of readout: (1) the smaller signals from light nuclei are measured using proportional counters. This type of detector gives a quite noise-free amplification of the weak signals from light nuclei and moreover, using charge- division and pad techniques, the coordinate in the non bending plane can be determined. (2) For heavy nuclei the charge resolution of the proportional counters deteriorates due to quenching effects, therefore these large signals are recorded with the anode strips.

The readout electronics of the anode strips is placed on the back of the strips and these are separated just by few centimeters from the proportional counters, which are operated at a gain of 1000 to 1500. Therefore a very good shielding between the two types of detectors is required (see Fig.4). In order to working out the already mentioned huge dynamic range, the electronic readout has been accordingly designed. In particular the signal coming from both the anode plane, the pad and the anode strips are readout by the above mentioned charge sensitive preamplifiers, whose gains have been chosen to be 33,10 and $1 \mathrm{pF}$, respectively, in order to match the corresponding charge range. 14-bit Flash ADC's digitise the signals coming directly from the preamplifiers. The 
output, generated at a rate of up to $40 \mathrm{MHz}$, is stored and processed by a system containing FPGA (Field Programmable Gate Array) and DSP (Digital Signal Processor) chips which will perform the necessary signal shaping as well as the determination of the collected charge and arrival time. On the same board fast digital and high-resolution analog circuits are operating without deterioration of the resolution. The upgraded detector, with its dedicated electronics system, is providing at the same time high resolution, a large dynamic range and multi-hit capability.

\section{The HADES TOF-Wall}

We also actively took part in the development of the front-end electronics of the HADES TOFWall $[8,9]$.

Timing is measured by an electronic chain which includes a constant fraction discriminator (CFD), followed by a logic active delay (LAD) and then by a time to digital converter (TDC). The CFDs are 16-fold Camac modules C808 from CAEN, specially modified in order to better suit the plastic and PMT signal features. As the time conversion is operated in common start mode, we need to delay the ECL outputs of discriminators by 350ns. Using cable delays was not viable, therefore we decided to design our own LAD, based on a very stable ramp discharging in constant current mode. The total delay can be varied continuously between 300 and $420 \mathrm{~ns}$ in each module, with an accuracy $\leq 5 \%$. The LAD, housed into a 32-fold NIM module, provides 32 ECL inputs and outputs, an analog multiplicity signal and a front panel trimmer in order to tune the delay value. The overall contribution of CFD and delay to the TOF resolution has been measured as $<100 \mathrm{ps}$.

We also designed our TDC, namely a 32- channel converter housed into a single VME unit. It implements 32 time-to-amplitude converter (TAC) sections and a multiplexer circuit, followed by a fast 12-bit ADC. Its total handling and conversion time for all the channels is o10 $\mathrm{ms}$ and the time resolution is $00.04 \%$ FWHM. Common start/stop mode, full scale range and individual thresholds are selected via software, as well as zero suppression and fast chained block transfer capabilities in order to speed up the readout.

The amplitude signal measurement is performed by an electronic chain made of a shaper and an amplitude to digital converter. The shaper, a 32- fold unit housed into a single width Camac station, has the task of reshaping and amplifying the signal from the PMT in order to match the input requirements of the ADC. Its gain can be selected via standard Camac functions. The ADC module, a 32-channel converter housed into a single VME unit, contains 32 linear gate and stretcher sections followed by a multi- plexer and an ADC. Once again the total conversion time is $<10 \mu s$, while the conversion gain is $1 \mathrm{mV} /$ channel. Rise-time protection, thresholds, zero suppression and chained block transfer are software selectable. Actually the TDC and ADC modules employ a common motherboard, hosting the logic circuitry and the true analog to digital conversion electronics, while the time or amplitude type characterization is done by fitting in one of two different daughter boards.

\section{Conclusions}

The scientific activity and the academic career of Ileana Iori gave us an unique opportunity to work in fundamental researches with very important achievements. With her enthusiasm and her 


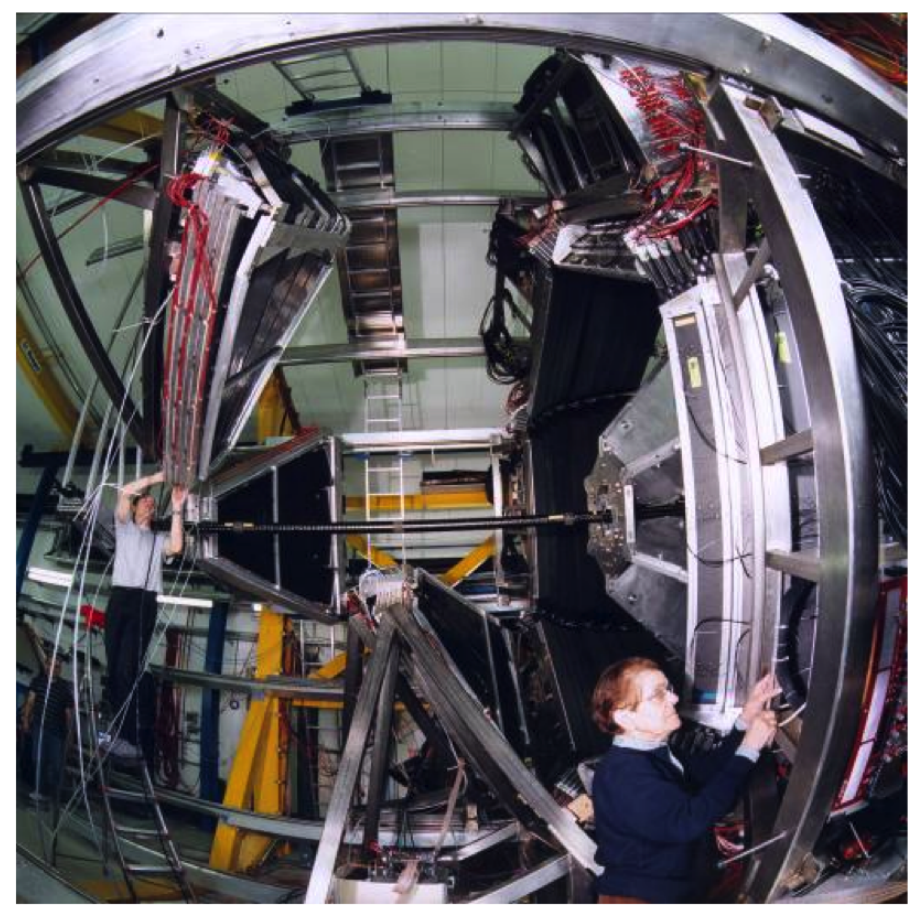

Figure 5: Ileana at work during the installation of the TOF-Wall at the HADES Spectrometer

strong recognized leadership, we were able to cover almost all of the most significant progresses in the field of detections, readout and data storage of nuclear signals in Heavy Ion Physics.

All the electronics team in Milano had the opportunity to have important interactions with high level nuclear laboratories around the world and to work with scientists and technologists of preeminent position.

In particular, it was for me a real privilege to collaborate with her for so a long period with unchanged enthusiasm and happiness.

Grazie ILEANA!

\section{References}

[1] I.Iori, et al., Nucl Instr and Meth A 325 (1993) 458.

[2] R .Bassini, et al., Nucl.Instr and Meth. A 305 (1991) 449.

[3] R.Bassini, et al., Nucl. Instr. and Meth. A327 (1993) 535.

[4] R.Bassini, et al., IEEE Trans. Nucl. Sci. NS-37 (6) (1990)

[5] U. Lynen, this conference proceeding.

[6] G. Raciti et al., Phys. Rev. Lett. 100 (2008) 192503.

[7] C. Sfienti et al., Proceeding of the XLI International Winter Meeting on Nuclear Physics Bormio (Italy) Ricerca Scientifica ed Educazione Permamente Supplemento n. 120 p. 323 (2003)

[8] P. Finocchiaro, this conference proceeding.

[9] R. Bassini, et al., IEEE Trans. Nucl. Sci. NS-45 (3) (1998) 858. 